\title{
Performance of Statistical Methods to Address Treatment Non-Adherence in Pragmatic Clinical Trials with Point-Treatment Settings: A Simulation Study
}

\author{
Md. Belal Hossain ${ }^{1, *}$, Lucy Mosquera ${ }^{2}$, and Mohammad Ehsanul Karim ${ }^{1,3}$ \\ ${ }^{1}$ School of Population and Public Health, University of British Columbia, Vancouver, Canada \\ ${ }^{2}$ Department of Statistics, University of British Columbia, Vancouver, Canada \\ ${ }^{3}$ Centre for Health Evaluation and Outcome Sciences, University of British Columbia, Vancouver, Canada \\ *E-mail: belal.hossain@ubc.ca
}

Received May 15, 2020

Accepted for publication June 1, 2020

Published online September 15, 2021

\begin{abstract}
Introduction: The instrumental variable (IV)-based methods (e.g., two-stage least square [2SLS], two-stage residual inclusion [2SRI], and nonparametric causal bound [NPCB]) can be used to address non-adherence in pragmatic trials. These methods require assumptions, e.g., exclusion restriction, although they are known to handle unmeasured confounding. The inverse probability-weighted per-protocol [IPW-PP] method is useful in the same setting but requires different assumptions (no unmeasured confounding). Although all these methods aim to address the same problem, comprehensive simulations to compare their performance are absent in the literature. We performed extensive simulations when (1) confounding is present, (2) confounder is unmeasured but exclusion restriction is met, (3) exclusion restriction is violated, and (4) non-adherence is one-sided and differential.
\end{abstract}

Method: We compared the performance in terms of bias, standard error (SE), mean squared error (MSE), and 95\% confidence interval coverage probability.

Results: For setting-1, IPW-PP outperforms IV-methods in terms of bias, SE, MSE, and coverage for < $80 \%$ non-adherence but produces high bias beyond that point. IPW-PP also has high biases, but 2SLS and 2SRI work well for setting-2. For setting-3, 2SLS and 2SRI perform the worst in all scenarios; IPW-PP produces unbiased estimates when necessary confounders are measured and adjusted. For setting-4, IPW-PP has less bias, but 2SLS and 2SRI have higher SE and MSE. NPCB has wider bounds in all scenarios. We also analyze a two-arm trial to estimate the effect of vitamin A supplementation on childhood mortality after addressing non-adherence.

Conclusion: We need to be cautious using the IPW-PP when non-adherence is very high or strong unmeasured confounding and should avoid using the IV methods when the exclusion restriction assumption is violated or high differential nonadherence. Since assumptions are different and often untestable for IPW-PP and IV methods, we suggest analyzing data using both methods for a robust conclusion. 